\title{
XPS-evidence for in-situ electrochemically-generated carbene formation
}

\author{
Pinar Aydogan Gokturk ${ }^{\mathrm{a}}$, Ulrike Salzner ${ }^{\mathrm{a}}$, László Nyulászi ${ }^{\mathrm{c}}$, Burak Ulgut ${ }^{\mathrm{a}, *}$, \\ Coskun Kocabas ${ }^{\mathrm{b}}$, Sefik Suzer ${ }^{\mathrm{a}, *}$ \\ a Department of Chemistry, Bilkent University, 06800 Ankara, Turkey \\ b Department of Physics, Bilkent University, 06800 Ankara, Turkey \\ ${ }^{\mathrm{c}}$ Department of Inorganic and Analytical Chemistry, Budapest University of Technology and Economics, Budapest, Hungary
}

\section{A R T I C L E I N F O}

\section{Article history:}

Received 4 January 2017

Received in revised form 27 February 2017

Accepted 6 March 2017

Available online 14 March 2017

\begin{abstract}
A B S T R A C T
Stable N-heterocyclic carbenes (NHC) are a class of compounds that has attracted a huge amount of interest in the last decade. One way to prepare NHCs is through chemical or electrochemical reduction of 1,3-disubstituted imidazolium cations. We are presenting an in-situ electrochemical X-ray Photoelectron Spectroscopy (XPS) study where electrochemically reduced imidazolium cations lead to production of stable NHC. The electroactive imidazolium species is not only the reactant, but also part of the ionic liquid which serves as the electrolyte, the medium and the electroactive material. This allows us to directly probe the difference between the parent imidazolium ion and the NHC through the use of XPS. The interpretation of the results is supported by both observation of reversible redox peaks in the voltammogram and the density functional theory calculations.
\end{abstract}

(C) 2017 Elsevier Ltd. All rights reserved.

\section{Introduction:}

Historically, stable carbene chemistry has not attracted much attention since it was always assumed that the stability of carbenes were too short-lived for any useful study. A seminal paper by Arduengo et.al. [1] resulted in a fundamental shift in this field by reporting that stable and "bottle-able" carbenes can be synthesized. The stability is achieved sterically by making use of bulky groups and electronically by using pi-donating, sigma withdrawing groups. The molecule reported (1,3-di-l-adamantylimidazol-2ylidene) contains a 5-membered imidazole ring, which is substituted with bulky adamantyl groups. This study was followed by a large body of work (recently reviewed by Hopkinson et. al. [2]), reporting synthesis of numerous molecules with the generic formula $\left(\mathrm{R}_{2} \mathrm{~N}\right) 2 \mathrm{C}$ :, where $\mathrm{R}$ can be aryl or alkyl groups. In a significant number of cases, the $\mathrm{R}$ groups contain nitrogen and are linked to form heterocyclic rings. This group of molecules are thus called N-heterocyclic carbenes (NHC).

Historically, carbenes had been synthesized in a number of different ways as reported by Wanzlick [3]. More recently, NHCs have most commonly been synthesized beginning with a 1 ,

\footnotetext{
* Corresponding author.

E-mail addresses: ulgut@fen.bilkent.edu.tr (B. Ulgut), suzer@fen.bilkent.edu.tr (S. Suzer).
}

3-disubstituted imidazolium salt and to generate the carbene via deprotonation using a strong base (see for example [4] and [5]). This method is reportedly used in organic and inorganic catalysis, where the carbene site is the crucial component of a number of important catalytic reactions as reviewed by W. A. Hermann [5]. It is also shown that electrochemical or chemical reduction of a 1,3disubstituted imidazolium salt under the right conditions leads to an NHC [6]. Chemically or electrochemically generated NHCs have been shown to be active catalysts for a number of reactions (see Feroci et. al. [7]).

One example of an electrochemical preparation of a stable carbene was reported by Gorodetsky et. al. [6]. A 1,3-disubstituted imidazolium salt was shown to turn into a carbene by reduction under electrochemical conditions. The formation of the stable carbene upon reduction of the imidazolium ion was shown by the emergence of a new electrochemical feature in the cyclic voltammogram.

Room temperature ionic liquids are a class of compounds that have appreciable ionic conductivities and very low vapor pressures at room temperature. This makes them uniquely suited as electrolytes for in-situ experiments under vacuum. These properties have been regularly exploited in experiments involving electrochemistry in vacuum systems [8,9]. Along these lines, we have recently reported another experiment studying the details of the formation of electrical double layer on a gold electrode using an 
ionic liquid [10]. 3,5-Substituted imidazolium derivatives with asymmetric substituents are very commonly used as cations of room temperature ionic liquids, mostly because of their stability and very low propensity to crystallize. Currently, room temperature ionic liquids involving 1,3-disubstituted imidazolium cations are commercially commonplace. Using ionic liquids based on imidazolium derivatives, it was claimed that NHCs can be electrochemically prepared, where it was indirectly shown via NMR spectroscopy that electrochemical reduction of a group of imidazolium derivatives yielded the corresponding NHC through the NHCs' catalytic activity in various organic reactions [11]. By following the products of the catalyzed reactions, the presence and amount of the NHCs were inferred. More recently, Feroci et. al. [12] reported cyclic voltammetric studies of thiazolium salts in the presence and the absence of acetate ions. Under the conditions that were used, the authors showed that NHCs are not stable unless acetate ions are incorporated into the mixture. They further presented voltammetric evidence of NHCs upon reduction of imidazolium systems. 1-ethyl-3-methylimidazolium acetate has already been extensively reported in the literature to have desirable properties for application in cellulose and chitin processing or carbon dioxide absorption ([13] and references therein). High basicity of the acetate anion helps stabilize the NHC upon reduction of the imidazolium cation.

X-ray photoelectron spectroscopy (XPS) is an established analytical tool that is not only used for quantitative chemical analysis since its inception, but also has been the platform for analyzing charging effects of various systems as reported by us $[14,15]$ and others $[16,17]$. In one example of NHC preparation using ionic liquids, Ir nanoparticles were employed to stabilize the carbene site. In that work, Bernardi et. al. [18] showed that adding Ir to a sample of ethyl-3-methylimidazolium ethyl sulfate (EMI. EtSO4), resulted in some oxidized Ir and formation of a new carbon species with lower electron binding energy. Using XPS, they were able to assign the new carbon signal to a carbene species that is interacting with the Ir nanoparticle surface that is resulting in a partially oxidized Ir species. A detailed in-situ synchrotron based XPS analysis was recently reported by Kruusma et al. on 1-ethyl-3methylimidazolium tertrafluoroborate [EMImBF4] deposited on molybdenum carbide derived carbon electrode while the electrode was under various negative biases to follow the electrochemical reduction products [19]. In the pristine EMImBF4 salt, the N1s peak has only one component, corresponding to the two equal nitrogen atoms on the imidazolium ring, bearing only one positive charge $(-\mathrm{N}=\mathrm{CH}-\mathrm{N}-)+$, with the corresponding binding energy of $402.7 \mathrm{eV}$. They reported that a second peak at a lower binding energy of $400.8 \mathrm{eV}$ appeared and grew in intensity as the electrode was polarized to higher negative values of up to $-2.40 \mathrm{~V}$ (vs. $\mathrm{Ag} / \mathrm{AgCl}$ reference electrode). In parallel, intensity changes in the corresponding $\mathrm{C} 1 \mathrm{~s}, \mathrm{~B} 1 \mathrm{~s}$ and $\mathrm{F} 1 \mathrm{~s}$ peaks were reported. They attributed these spectral changes to electrochemical reduction as well as to decomposition products. In addition, they reported on observation of shifts in the position of the binding energies as a function of the potentials applied to the electrode(s), similar to those reported earlier by Foelske-Schmitz et al., where a potentiostatically controlled DC bias was imposed using an electrochemical set-up and recording XPS [20-22]. The unique ability of XPS to yield chemically specific information about local charging allows in depth studies of charge accumulation. This has been particularly tunable with the application of external electrical bias onto the sample with respect to the instrument ground [23,24].

In the present report, XPS has been used to gather oxidation state information of individual atoms. We are reporting on an experiment where we simultaneously generate carbenes electrochemically and detect, with strong experimental and theoretical evidences, the formation of a carbene species through the changes of the chemical environment of neighboring nitrogen atoms. To our knowledge, this is the first report of reversible carbene electrochemistry as evidenced by small peak separation in the voltammogram coupled to the reduction charge being equal to the oxidation charge.

\section{Results and Discussion}

Fig. 1 shows the chemical structure and a survey spectrum of the ionic liquid. The XP spectrum of the EMImAc includes photoelectron peaks for nitrogen $1 \mathrm{~s}$, oxygen $1 \mathrm{~s}$ and carbon $1 \mathrm{~s}$ electrons. The nitrogen $1 \mathrm{~s}$ region is specifically the region we will be following, as that region is going to develop the readily observable changes due to the carbene formation. The carbon region, which should also develop relevant changes, is much harder to decode unequivocally as there are more species of carbon at different binding energies. However, as will show below, the region also provides relevant and supporting information.

As seen in the N1s region, as prepared sample shows only a single peak in the spectrum that corresponds to the two equivalent nitrogen atoms of the imidazolium ring, carrying overall one unit of positive charge, at a binding energy of $402.2 \mathrm{eV}$, which is similar with the previous studies on imidazolium based ionic liquids [2326]. The fitting of $\mathrm{C} 1 \mathrm{~s}$ region is also illustrated in Fig. 1 for EMImAc. The contribution to C1s envelope comes both from the EMIm cation and the acetate ions. The specific carbons are numbered on the structure that is drawn to illustrate the various contributions. For all carbons a Lorentzian/Gaussian mix (30\% Gaussian) peak shape was used. We have adopted the binding energy values used in Ref. [23] for the C1s peaks of the cation and the guidance outlined in Ref. [27] for others. Accordingly, $C_{2}$ and $C_{5}$, as well as $C_{3}$ and $C_{4}$ are assigned to be equivalent. Therefore, the area for these two peaks were constrained to be twice as much as the other carbons. $C_{6}$ is the aliphatic carbon that the binding energy was shifted to $285.00 \mathrm{eV}$ to correct for charging effects [17]. The fact that, features due to the underlying silicon is not observed on the survey spectrum, indicates that the thickness of the ionic liquid on the region worked on is thicker than the average scattering length of the photoelectrons, which is known to be $5-10 \mathrm{~nm}$.

1-Ethyl-3-Methyl Imidazolium Acetate

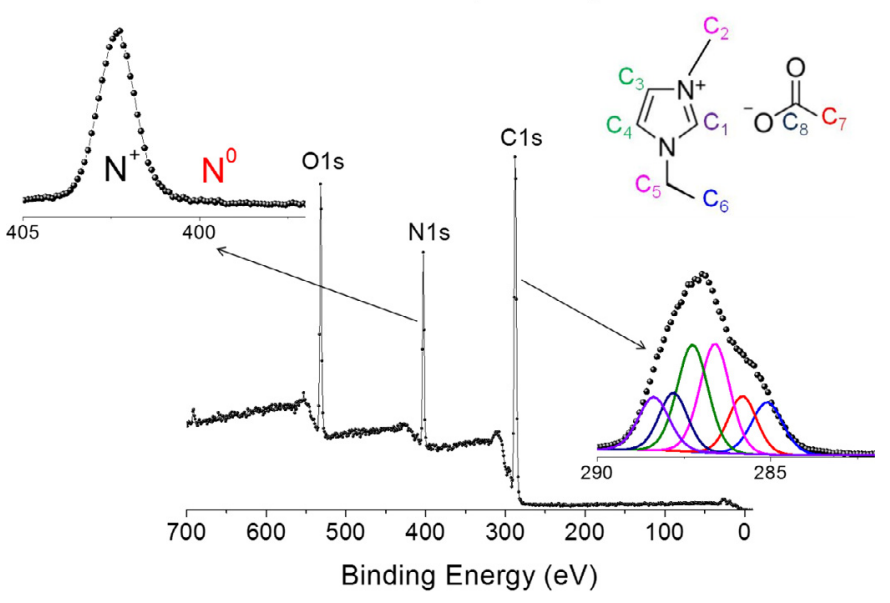

Fig. 1. A Survey XP spectrum of the EMImAc together with the N1s and C1s levels recorded at a higher resolution. The $\mathrm{N} 1 \mathrm{~s}$ region consists of a single peak assignable to the two equivalent nitrogen atoms of the cyclic structure, but the complex C1s region is fitted to 8 peaks two of which have been fitted to two equivalent carbons as indicated in the inset. 


\subsection{Bias Shifts in XPS}

As the main emphasis of this work is to demonstrate that, by application of an external voltage bias electrochemical reduction is achieved to yield stable NHC, where XPS is utilized to analyze and at the same time to follow the process, a warning needs to be introduced at this point. Application of external bias in XPS has also been shown to cause differential charging in various samples and devices [14-17]. More recently, we have reported on a symmetric coplanar capacitor geometry with an ionic liquid as the conducting medium in an XPS setting, where the details of the surprising longrange charging/discharging effects of the double layer, along with its kinetic behavior were explained and modeled [10]. This information was harvested through analyses of XPS peak shifts of certain elements only after imposing DC and/or AC electrical biases to the sample. However, as was also cautioned in Ref. [27], great care must be exercised to extract the correct information, since the very same bias can introduce spectral artifacts as well, as shown in Figure S1 in the Supporting Information (SI) section. These shifts, caused by applied potential, can easily be mistaken for chemical shifts, as the applied potential can shift different species differently, as a result, different peaks may overlap or get separated, depending on induced local charges. Therefore, in order to judge the identity of different chemical species, it is best to compare data that is taken with grounded samples in order to mitigate any adverse effects of potentially non-uniform bias effects, as depicted in Fig. 2.

It is clearly visible in Fig. 2 that even after the electrodes are grounded, two separate $\mathrm{N} 1 \mathrm{~s}$ peaks prevail. This proves, without any doubt that a new chemical species is generated and the new signal is not due to any partial charging or heterogeneous electric field effects (see Figure S1 in the SI section). However, the C1s exhibits slight broadening in the high energy side under $-3 \mathrm{~V}$ due most probably to partial charging.

\subsection{Electrochemical Reduction}

Once enough bias is applied, a reduction is expected on the negatively biased electrode that would lead to carbene formation. Under extended periods of applied bias, the carbene species can be shown to form on either electrode depending on the polarity of the applied potential. As Fig. 3 shows, throughout 8 hour periods, we can observe a new N1s signal formed on either $-3 \mathrm{~V}$ or $3 \mathrm{~V}$ applied across the ionic liquid sample. This new signal that forms with a binding energy that is $\sim 2.5 \mathrm{eV}$ lower than the binding energy of the $\mathrm{N} 1 \mathrm{~s}$ of the imidazolium ring is attributed to the nitrogen atoms that are part of the overall neutral NHC. When a positive bias is applied to the gold electrode, the silicon electrode is effectively at $-3 \mathrm{~V}$ vs. the gold wire. This voltage is enough for the silicon electrode to reduce the imidazolium cation, leading to the

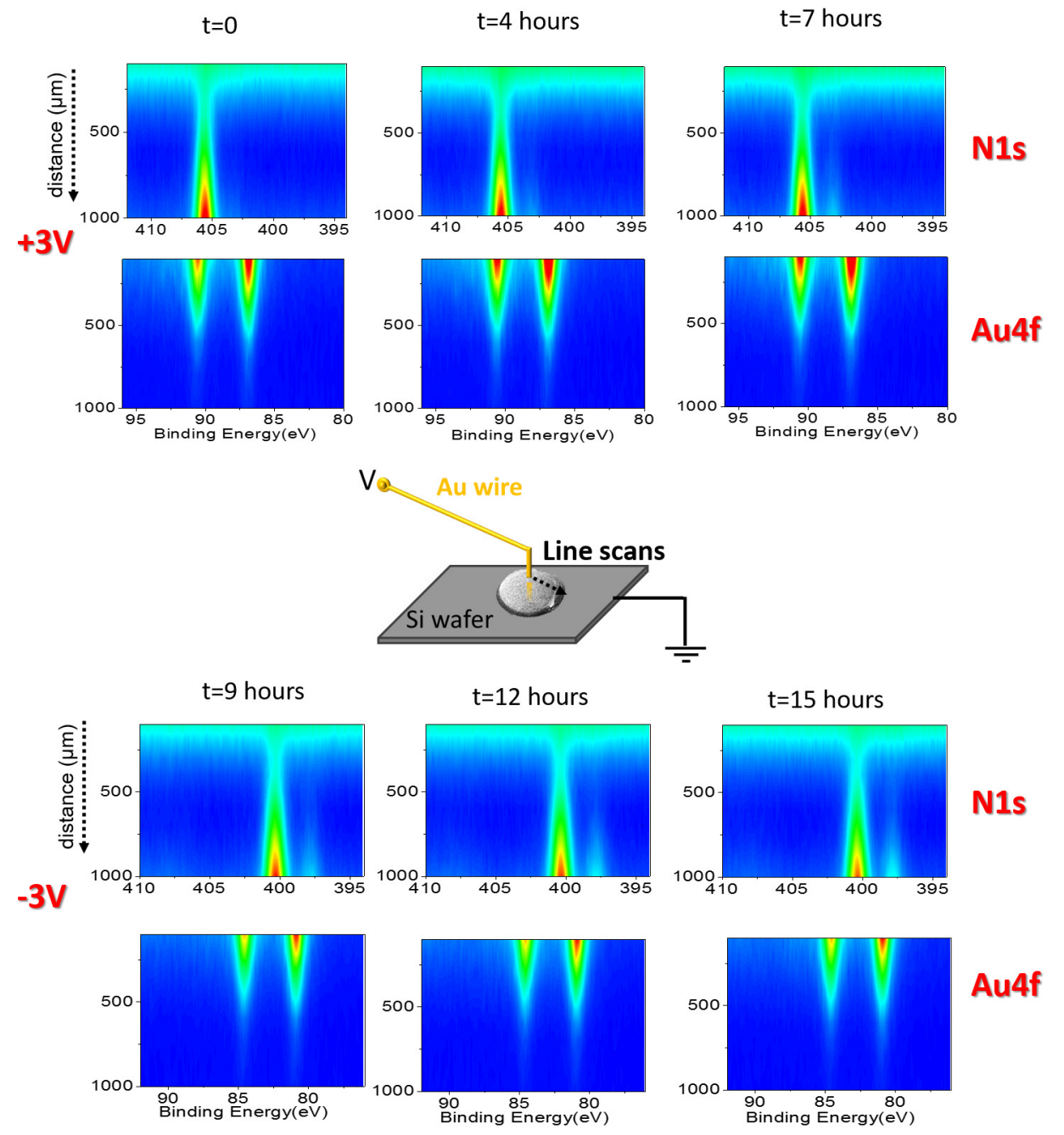

Fig. 2. The N1s and $\mathrm{C} 1 \mathrm{~s}$ regions after electrochemical reduction and under $+3,0$ (ground) and $-3 \mathrm{~V}$ external bias. 
formation of the carbene. When a negative bias is applied to the gold electrode, the reduction happens on the gold electrode, and the carbene N1s signal becomes more visible near the gold wire. Both of these expectations are indeed verified by results displayed in the figure, in which the first appearance of the new peak is in the vicinity of the Si-electrode and moves up to the Au-electrode in time, and only after switching the bias to $-3 \mathrm{~V}$ the intensity grows near that electrode. In parallel oxidized $\mathrm{Si}$ moieties $\left(\mathrm{SiO}_{\mathrm{x}}\right)$ are also produced within the IL medium (not shown).

In order to justify our argument that the new N1s peak is due to a different chemical species and not due to heterogeneous electric potential effects, the XPS measurements are repeated at the end while both the electrodes are grounded as was shown in Fig. 2.

In order to further show that these changes cannot be explained by experimental artefacts like X-ray beam damage, the control experiment of extended beam exposure was performed. 20 hours of X-Ray exposure yields no noticeable change in the $\mathrm{C} 1 \mathrm{~s}, \mathrm{O} 1 \mathrm{~s}$ and $\mathrm{N} 1 \mathrm{~s}$ regions of the photoelectron spectrum. This data is shown in the supporting information as Figure S2.

\subsection{Chemical and Electrochemical Shifts Revealed by XPS}

The strength of XPS has traditionally been attributed to its ability to differentiate the bonding information of the analyzed atoms via the so-termed chemical shifts. Equally important is its ability to reflect the electrical potential of the medium, since the measured kinetic energy of the photoelectrons are directly affected. Therefore, it is of utmost importance to disentangle them for correct interpretation of the results. When the N1s region of the EMImAc before and after electrochemical reduction and under different bias applications (Fig. 2) is examined the new peak at $\sim 2.5 \mathrm{eV}$ lower energy can be assigned to a neutral-N (imide) within the structure.

Upon application of $3 \mathrm{~V}$ external bias all the peaks of both the $\mathrm{N} 1 \mathrm{~s}$ and $\mathrm{C} 1 \mathrm{~s}$ regions undergo a shift of +2.4 and $-2.5 \mathrm{eV}$ shifts, respectively, due to the voltage developed within the IL medium under the potential stress. The absolute magnitude of $2.5 \mathrm{eV}$ indicates that $0.5 \mathrm{~V}$ drops across the $\mathrm{Au}$ electrode/electrolyte interface and $2.5 \mathrm{~V}$ on the Si electrode/electrolyte interface. Since the same absolute amount of excess charge exists on both electrodes, the voltage drop across the interface is inversely proportional to the double layer capacitance on the respective electrodes $\left(V_{\text {double layer }}=q_{\text {double layer }} / c_{\text {double layer }}\right)$. This indicates that the overall effective capacitance (and thus the contact area) ratio of the

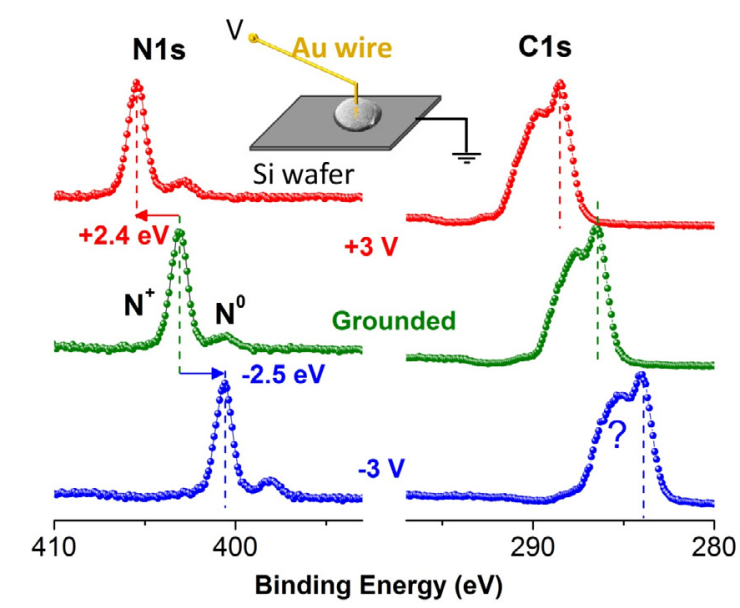

Fig. 3. XP Spectra of theN1s and Au4f regions along the line designated under $+3 \mathrm{~V}$ and $-3 \mathrm{~V}$ Bias and in different periods. two electrodes is 1:5. It can be concluded that the effective contact area between the Au electrode and the ionic liquid is 5 times greater than the contact area between $\mathrm{Si}$ electrode and the ionic liquid. However, the uniformity of this shift is not always granted, since it is possible that depending on the type (two electrode or three electrode) and the chemical nature of the electrochemical set-up used, the size (small spot vs. large one) the make-up of the analyzed region, and the amount and uniformity of the current flow, non-uniform shifts could develop.

These potential profiles that are prone to non-uniformities could lead to non-uniform shifts in the binding energies. The easiest way to circumvent this is to record data after removal of the external potential stresses and allow ample time before recording the spectrum to ensure complete discharge. Doing the experiment with grounded electrodes at the end of the potential application ensures that any new peaks that are observed are due to new chemical species and not due to any non-uniform potential effects.

\subsection{In-situ Cyclic Voltammograms and XPS Recording}

For further understanding of the electrochemistry in the two electrode geometry, cyclic voltammetry experiment was performed starting at $0 \mathrm{~V}$ and sweeping to $-2 \mathrm{~V}$ and back at a sweep rate of $1 \mathrm{~V} / \mathrm{s}$. The data is shown in Fig. 4(d). The voltammogram shows a clear redox couple that we attribute to the redox reaction that leads to formation of the carbene in a reversible manner. Reduction of the imidazolium cation leads to the formation of the relevant NHC that can get re-oxidized back to the imidazolium. This signal is chemically reversible as shown by the fact that the area underneath both peaks are the same. This is strong evidence that the carbene that is formed is stable throughout the time scale of the experiment. This is in strong contrast to the previous reports of reduction of imidazolium derivatives to produce carbene species ([12]) where the formed carbene does not show any reversibility. This shows that inside the XPS chamber where water vapor or oxygen gas is minimized, the electrochemically formed carbene is stable. In the two electrode geometry, it is hard to judge electrochemical reversibility since $\Delta \mathrm{E}_{\text {peak }}$ needs to be evaluated. Electrochemical reversibility can be simply measured by an accurate measurement of $\Delta \mathrm{E}_{\text {peak }}$. However, in the two electrode geometry, the resistance of the ionic liquid is not compensated and therefore contributes significantly to the potentials and thus the $\Delta \mathrm{E}_{\text {peak }}$ that are measured.

In order to follow the formation of the carbene species via XPS in real time, a much slower sweep rate $(1 \mathrm{mV} / \mathrm{s})$ needed to be used in order to develop the necessary amount of material to get appreciable XPS signal. At these sweep rates, the faradaic current is below the noise level, so the linear sweep voltammogram (not shown) does not show any appreciable features, but the XP spectra develop the N1s peak that is attributed to the NHC. This peak grows as the potential is swept more negative. This is not followed on the reverse sweep as the data gets complicated with effects of the stable carbene species diffusing away from the electrode (data not shown). The XPS result of this experiment is shown in various forms in Fig. 4 (a,b,c). Fig. 4a shows a contour map of the XP spectra at various potentials. As the potential is swept more negative, in addition to the overall waveform shifting to lower binding energies, a new peak starts forming at $\sim 2.5 \mathrm{eV}$ lower binding energies. This is more prominently shown in Fig. $4 \mathrm{~b}$ where the green spectrum shows a slice from $-2 \mathrm{~V}$ applied potential. This spectrum clearly shows the peak that is attributed to the NHC designated as $\mathrm{N}^{0}$. The details of this formation is summarized in Fig. $4 \mathrm{c}$ where the ratio of the peak intensities are plotted as a function of the applied bias. It is clear that the NHC amount increases as the potential is swept more negative. 

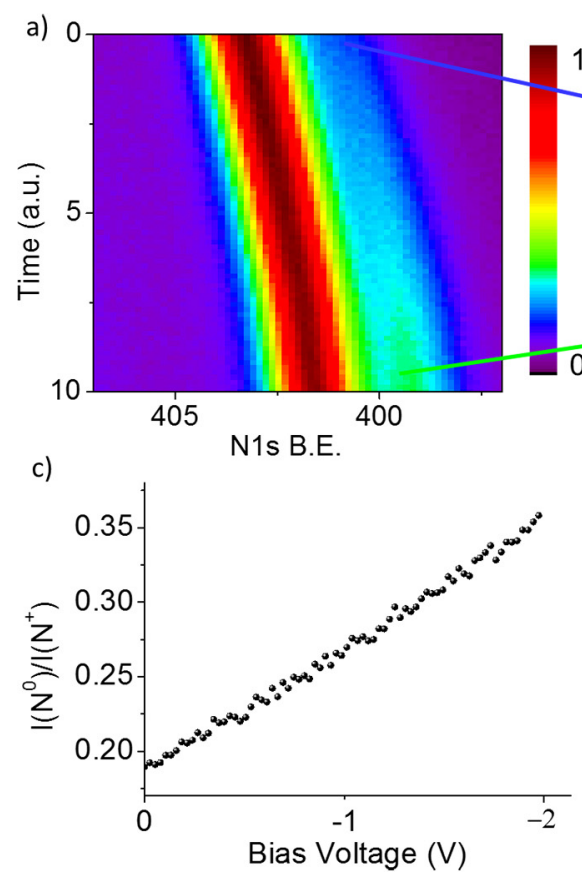

b)

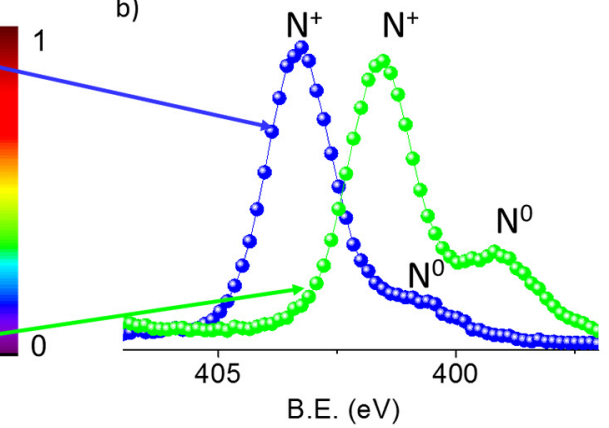

d)

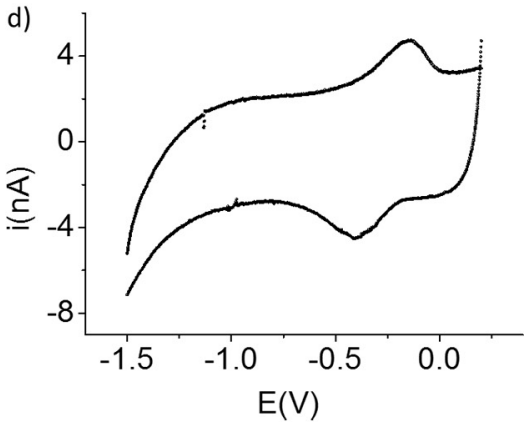

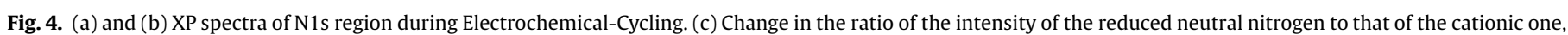
with the applied potential. (d) Two electrode cyclic voltammagram of the system.

Table 1

Experimentally determined B.E. positions of the C1s and N1s peaks together with calculated shifts after carbene formation.

\begin{tabular}{|c|c|c|c|c|c|c|c|}
\hline & $\mathrm{C}_{1}$ & $\mathrm{C}_{2}$ & $\mathrm{C}_{3}$ & $\mathrm{C}_{4}$ & $\mathrm{C}_{5}$ & $\mathrm{C}_{6}$ & $\mathrm{~N}$ \\
\hline EMImAc binding energies(eV) & 288.38 & 286.58 & 287.28 & 287.28 & 286.58 & 285.00 & 402.20 \\
\hline Calculated shifts $(\mathrm{eV})$ & -2.45 & -0.72 & -1.07 & -1.03 & -0.35 & -0.24 & $-1.53^{*}$ \\
\hline
\end{tabular}

*Average value for the two nitrogen atoms. Details are given in supplementary information.
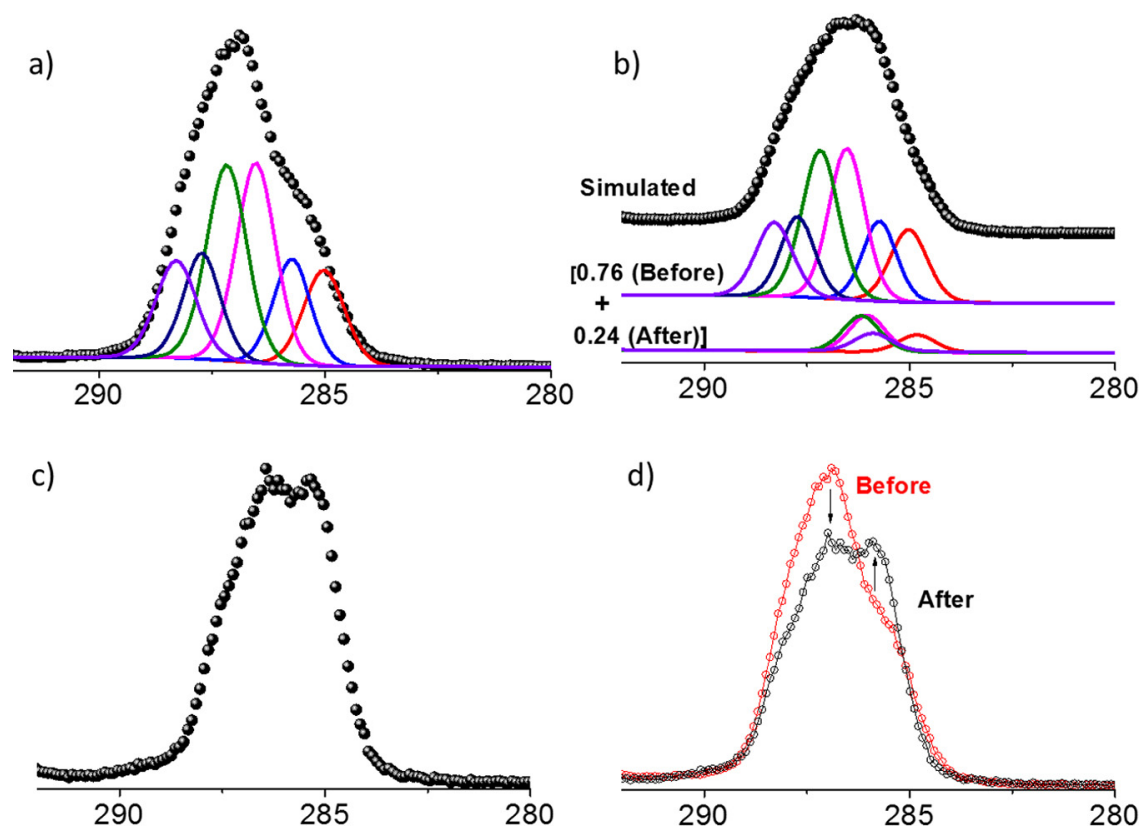

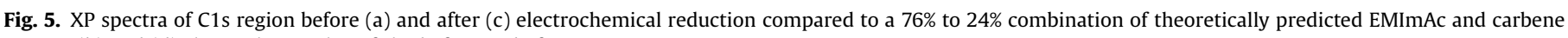
spectra (b) and (d) shows the overlay of the before and after spectra. 


\section{Discussions}

After the in situ electrochemical reduction of EMImAc ionic liquid under external bias, not only the N1s region but also $\mathrm{C} 1 \mathrm{~s}$ region developed relevant changes, i.e. an increase in the intensity of low binding energy components of the C1s envelope, see Fig. 2. Similar changes were also observed in previous studies and attributed to increasing intensity of aliphatic carbon peaks [C5, C6] [19]. Additionally, from the intensity ratio of the cationic to the neutral N1s components we determine that $24 \%$ of the nitrogen atoms undergo electrochemical reduction within the analyzed region.

In order to understand the changes in C1s spectrum after the electrochemical reaction, we calculated $1 \mathrm{~s}$ binding energies and the corresponding shifts. The shifts and experimental EMImAc binding energies are collected in Table 1. The details of the calculation and the calculated binding energies are shown in SI along with a discussion of the calculation results.

For all imidazolium carbon atoms theory predicts a decrease in binding energy which is strongest for the carbene carbon and weakens with distance from the carbene center as the overall positively charged imidazolium is now reduced to an overall neutral carbene. Binding energies of the carbon atoms of the acetate anion increase as the proton is accepted. Because the acetic acid formed during the reaction probably diffuses away or evaporates away from the carbene, the observed carbon peak after reduction may consist of the carbene carbon peaks only. High resolution C1s spectra of IL before and after the electrochemical process are compared with theoretical 1s shifts in Fig. 5. For the simulated shifts (Fig. 5b), the EMImAc and carbene peaks (without the acetic acid carbons) are combined with the weights of $76 \%$ and $24 \%$. These weights are experimentally determined using the ratio of the peak areas of the $\mathrm{N} 1 \mathrm{~s}$ signal. Although the simulated and experimental spectra are not identical, the intensity increase in the lower binding energy region is reproduced. This supports the experimental findings that suggest that the observed spectrum is a combination of EMImAc and carbene peaks, and it is clear that a contribution from a higher binding energy carbon is getting traded for a lower binding energy carbon (Fig. 5d). But, it should also be reminded that over the long term XPS measurements, adventitious carbon could have been accumulated, which might also be contributing to the low energy $(\sim 285.0 \mathrm{eV})$ component of the composite peak [27].

The cyclic voltammetry data inside the XPS chamber show that the substituted imidazolium cation species can get reduced to the related carbene species in a chemically reversible manner. This reversibility shows that in the absence of any oxygen and water, the carbene is stable within the time-scales of the experiment.

\section{Conclusions}

Reversible carbene electrochemistry was observed inside an XPS chamber. The new N1s peak with the binding energy of $399.8 \mathrm{eV}$ generated via electrochemical means is assigned to the reduced $\mathrm{N}$ atoms of the NHC. Electrochemical and XPS evidences indicate that reduction of imidazolium cations in the presence of acetate ions leads to reversible formation of NHCs, which is furher supported by calculations.

\section{Experimental Section}

1-Ethyl-2-methyimidazolium acetate [EMIMAc] was purchased from Sigma-Aldrich. Electrochemical experiments, along with the photoelectron spectroscopy were collected using a Si substrate that was connected to the instrument ground with a drop of ionic liquid formed on the polished side. A gold wire, was connected to the sample holder for electrical connection and was put into physical contact with the ionic liquid drop. XPS data were collected using a Thermo Fisher K-Alpha X-ray Photoelectron Spectrometer with a monochromatized photon energy of $1486.6 \mathrm{eV}$ for all measurements. In-situ electrochemical measurements were performed using a Keithley 2400 Source-meter that is driven by an inhouse written LabView Software in the controlled potential mode. The potential was programmed while the currents were measured every $1 \mathrm{~ms}$. The ground of the spectrometer was connected to the ground of the Keithley Source-Meter. This ensured that no ground loops existed between various instruments.

\section{Appendix A. Supplementary data}

Supplementary material related to this article can be found, in the online version, at http://dx.doi.org/10.1016/j. electacta.2017.03.053.

\section{References}

[1] A.J. Arduengo III, R.L. Harlow, M. Kline, JACS 113 (1991) 361.

[2] M.N. Hopkinson, C. Richter, M. Schedler, F. Glorius, Nature 510 (2014) 485.

[3] H.W. Wanzlick, Angew. Chem. Intl. Edit. 1 (2) (1962) 75.

[4] A.J. Arduengo III, R. Krafczyk, R. Schmutzler, Tetrahedron 55 (1999) 14523.

[5] W.A. Hermann, Angew. Chem. Intl. Ed. 41 (2002) 1290.

[6] B. Gorodetsky, T. Ramnial, N.R. Branda, J.A.C. Clyburne, Chem. Comm 17 (2004) 1972.

[7] M. Feroci, I. Chiarotto, M. Orsini, R. Pelagalli, A. Inesi, Chem. Comm 48 (2012) 5361.

[8] A. Foelske-Schmitz, D. Weingarth, R. Kötz, Echem. Acta 56 (2011) 1032110331.

[9] R. Wibowo, L. Aldous, R.M.J. Jacobs, N.S.A. Manan, R.G. Compton, Chem. Phys Lett. 509 (2011) 72-76.

[10] M.T. Camci, P. Aydogan, B. Ulgut, C. Kocabas, S. Suzer, Phys. Chem. Chem. Phys. 18 (2016) 28434-28440.

[11] I. Chiarotto, M. Feroci, G. Sotgiu, A. Inesi, Eur. J. Org. Chem (2013) 326-331.

[12] M. Feroci, I. Chiarotto, F. D’Anna, L. Ornano, C. Rizzo, A. Inesi, Electrochem. Comm 67 (2016) 55-58.

[13] Z. Kelemen, B. Péter-Szabó, E. Székely, O. Hollóczki, D.S. Firaha, B. Kirchner, J. Nagy, L. Nyulászi, Chem. Eur. J. 20 (2014) 13002-13008.

[14] H. Sezen, S. Suzer, Thin Solid Films 534 (2013) 1-11.

[15] C. Kocabas, S. Suzer, Anal. Chem. 85 (2013) 4172-4177.

[16] B. Bozzini, M. Amati, L. Gregoratti, M.K. Abyaneh, M. Prasciolu, A.L. Trygub, M. Kiskinova, J. Phys. Chem. C 116 (2012) 23188-23193.

[17] I.J. Villar-Garcia, E.F. Smith, A.W. Taylor, F. Qiu, K.R.J. Lovelock, R.G. Jones, P. Licence, Phys. Chem. Chem. Phys. (2011) 2797.

[18] F. Bernardi, J.D. Scholten, G.H. Fecher, J. Dupont, J. Morais, J. Chem. Phys. Let. 479 (2009) 113-116.

[19] J. Kruusma, A. Tonisoo, R. Parna, E. Nommiste, I. Tallo, T. Romann, E. Lust, Electrochem. Acta 206 (2016) 419-426.

[20] A. Foelske-Schmitz, D. Weingarth, H. Kaiser, R. Kötz, Electrochem. Commun 12 (2010) 1453-1456.

[21] A. Foelske-Schmitz, P.W. Ruch, R. Kötz, J. Electron Spectrosc. Relat. Phenom 182 (2010) 57-62

[22] D. Weingarth, A. Foelske-Schmitz, A. Wokaun, R. Kötz, Electrochem. Commun 13 (2011) 619-622.

[23] E.F. Smith, F.J. Rutten, I.J. Villar-Garcia, D. Briggs, P. Licence, Langmuir 22 (2006) 9386.

[24] I.J. Villar Garcia, K.R.J. Lovelock, S. Men, P. Licence, Chem. Sci. (2014) 2573.

[25] S. Men, D.S. Mitchell, K.R.J. Lovelock, P. Lience, ChemPhysChem (2015) 2211.

[26] K.R.J. Lovelock, E.S. Smith, A. Deyko, I.J. Villar-Garcia, P. Licence, R.G. Jones, Chem. Commun. (2007) 4866.

[27] K.R.J. Lovelock, I.J. Villar-Garcia, F. Maier, H.-P. Steinruck, P. Licence, Chem. Rev (2010) 5158. 